\title{
Influência da Realidade Virtual e Aumentada no tratamento da dor do membro
}

\section{fantasma: Revisão integrativa}

\author{
Influence of Virtual and Increased Reality in the treatment of ghost member pain: Integrative \\ review
}

Influencia de la Realidad Virtual Y Aumentada en el tratamiento del dolor en el miembro

fantasma: Revisión integrativa

Recebido: 28/06/2021 | Revisado: 05/07/2021 | Aceito: 09/07/2021 | Publicado: 21/07/2021

\author{
Eloisa Cesário Fernandes \\ ORCID: https://orcid.org/0000-0002-9330-1245 \\ Universidade do Estado do Rio Grande do Norte, Brasil \\ E-mail: eloisa_cesario@hotmail.com \\ Mauro Bezerra do Nascimento Júnior \\ ORCID: https://orcid.org/0000-0002-3116-1013 \\ Universidade do Estado do Rio Grande do Norte, Brasil \\ E-mail: maurojunior@alu.uern.br \\ Francisco Emilio Simplício de Souza \\ ORCID: https://orcid.org/0000-0001-6648-5141 \\ Universidade do Estado do Rio Grande do Norte, Brasil \\ E-mail: emiliosimplicio@uern.br \\ Bárbara Rebecca Fernandes de Farias \\ ORCID: https://orcid.org/0000-0001-8646-5893 \\ Universidade do Estado do Rio Grande do Norte, Brasil \\ E-mail: barbararebecca@hotmail.com \\ Ellany Gurgel Cosme do Nascimento \\ ORCID: https://orcid.org/0000-0003-4014-6242 \\ Universidade do Estado do Rio Grande do Norte, Brasil \\ E-mail: ellanygurgel@uern.br \\ Vanessa de Paiva Melo \\ ORCID: https://orcid.org/0000-0001-9345-2222 \\ Universidade do Estado do Rio Grande do Norte, Brasil \\ E-mail: melop.vanessa@gmail.com
}

\begin{abstract}
Resumo
Objetivo: Avaliar os tratamentos de tecnologia virtual e aumentada no manejo da dor do membro fantasma. Metodologia: A busca da literatura científica foi realizada nas bases de dados Pubmed, Scielo, Lilacs, Web of Science, Scopus e CINAHL, de estudos primários publicados no período de 2015 a 2020, com indivíduos com diagnóstico de dor em membro fantasma. Foram identificados 380 artigos, os quais após os critérios de elegibilidade resultaram em 16 estudos. Resultados: Estudos com as referidas realidades apresentaram diminuição da intensidade e percepção da dor no membro, a partir do questionário de dor e as pesquisas associadas com a realidade aumentada, os indivíduos melhoraram em suas atividades diárias bem como na qualidade do sono. Conclusão: A realidade virtual e aumentada apresenta-se como uma estratégia eficiente no tratamento, contudo, são necessários estudos com amostras e tempo maior para verificar a eficiência em longo prazo.
\end{abstract}

Palavras-chave: Terapia de realidade virtual; Realidade aumentada; Membro fantasma.

\begin{abstract}
Objective: To evaluate virtual and augmented technology treatments in the management of phantom limb pain. Methodology: The search for scientific literature was carried out in the databases Pubmed, Scielo, Lilacs, Web of Science, Scopus and CINAHL, of primary studies published in the period from 2015 to 2020, with individuals diagnosed with phantom limb pain. 380 articles were identified, which after the eligibility criteria resulted in 16 studies. Results: Studies with the referred realities showed a decrease in the intensity and perception of pain in the limb, based on the pain questionnaire and the researches associated with the augmented reality, the individuals improved in their daily activities as well as in the quality of sleep. Conclusion: Virtual and augmented reality presents itself as an efficient treatment strategy, however, studies with samples and more time are needed to verify long-term efficiency.
\end{abstract}

Keywords: Virtual reality therapy; Augmented reality; Phantom limb. 


\section{Resumen}

Objetivo: Evaluar tratamientos de tecnología virtual y aumentada en el manejo del dolor del miembro fantasma. Metodología: La búsqueda de literatura científica se realizó en las bases de datos Pubmed, Scielo, Lilacs, Web of Science, Scopus y CINAHL, a partir de estudios primarios publicados de 2015 a 2020, con individuos diagnosticados de dolor de miembro fantasma. Se identificaron 380 artículos, que luego de los criterios de elegibilidad resultaron en 16 estudios. Resultados: Estudios con estas realidades mostraron disminución de la intensidad y percepción del dolor en la extremidad, a partir del cuestionario de dolor y la investigación asociada a la realidad aumentada, los individuos mejoraron en sus actividades diarias así como en la calidad del sueño. Conclusión: La realidad virtual y aumentada se presenta como una estrategia eficiente en el tratamiento, sin embargo, se necesitan estudios con muestras y mayor tiempo para verificar la eficiencia a largo plazo.

Palabras clave: Terapia de realidad virtual; Realidad aumentada; Miembro fantasma.

\section{Introdução}

A realidade aumentada (RA) é uma tecnologia emergente que ajuda na compreensão de muitas situações e permite uma associação entre o mundo virtual e o mundo real, por meio de sofisticados programas que geram uma imagem integral e aumenta a cena virtual com o real (Uzun, 2009; Ayoub, 2019). Além disso, não tem limite específico de aplicabilidade, podendo ser utilizada para deixar jogos cada vez mais interativos, para melhorar os processos da medicina, como por exemplo, realização de cirurgias remotas. Tanto a RA como a realidade virtual (RV) têm evoluído com uma presença crescente no atendimento clínico, treinamento cirúrgico e tratamento de algumas síndromes, como é o caso do uso da realidade virtual e aumentada para o tratamento da dor do membro fantasma e acompanham a evolução das tecnologias (Cao, 2019; Nunzio, 2018).

Iniciado pela primeira vez por Jaron Lanier em 1986 para se referir a uma tecnologia 3D que utiliza um computador, monitor montado na cabeça e controladores equipados com pelo menos um rastreador de posição (Kim, 2017), o termo realidade virtual fornece plataformas seguras e flexíveis para a análise de várias regiões anatômicas do corpo durante exame, diagnóstico, planejamento e treinamento cirúrgico, além de auxiliar em algumas terapias (Ayoub, 2019; Akbulut, 2018).

Pode-se definir membro fantasma como a experiência de ter amputado algum membro do corpo e o mesmo se apresentar como se existisse, fazendo com que o paciente tenha sensações de membros fantasmas, devido as várias impressões da presença daquele membro. E a dor do membro fantasma pode ser caracterizada como uma dor em algum membro do corpo amputado, manifestada em diversas formas como ardor, aperto, compressão ou até mesmo uma dor intensa e frequente no membro ausente (Akbulut, 2019; Demidoff, 2007).

Estudos relatam que há uma prevalência de 80-85\% de pessoas que sofrem com essa alteração (Akbulut, 2019). E que a dor pode aparecer logo no início da amputação do membro, mas que pode surgir semanas, meses ou até anos depois, estando localizada geralmente na extremidade distal do membro fantasma (Demidoff, 2007).

Tendo em vista que a dor do membro fantasma pode afetar negativamente a qualidade de vida da população e que essa é uma condição importante que necessita de assistência à saúde, o presente artigo busca na literatura os possíveis tratamentos associados com base na tecnologia avançada da realidade virtual e aumentada, como forma de proporcionar melhoras para os indivíduos que sofrem com essa alteração.

\section{Metodologia}

Trata-se de uma revisão integrativa com coleta de dados realizada a partir de fontes primárias, por meio de levantamento bibliográfico na literatura. A pergunta científica norteadora da revisão, baseada no acrônimo PICO (Paciente, Intervenção, Comparação e Resultado/desfecho do estudo), foi definida como: A realidade virtual/aumentada influencia no tratamento do membro fantasma? 
Foram realizadas buscas e elencados, a partir de termos que constam na Medical Subject Headings (MESH) e nos Descritores em Ciências da Saúde (Decs), os seguintes descritores, respectivamente: "virtual reality", "augmented reality", "phanton limb"; "realidade virtual", "realidade aumentada", "membro fantasma".

Idealizando uma busca abrangente combinaram-se os três descritores do Decs, utilizando os operadores booleanos “OR" e "AND”, que gerou a estratégia de busca: "(Virtual reality OR Augmented Reality) AND phantom limb". A estratégia foi utilizada nas bases de dados: PubMed, SciELO, LILACS, Web of Science, Scopus e CINAHL, buscando os artigos de estudo primário publicados nessas plataformas no intervalo dos últimos 5 anos.

Para a seleção dos artigos foram pré-estabelecidos como critérios de inclusão os artigos publicados em português, inglês ou espanhol indexados nas bases de dados supracitadas e disponíveis de forma gratuitamente no Portal de Periódicos Capes. Como critérios de exclusão, estabeleceu-se que estariam descartados todos os estudos que não apresentassem relação com a temática de interesse, não fossem estudos primários e que fossem classificados como literatura cinzenta.

Seguindo os preceitos dos critérios de inclusão e exclusão, as buscas nas bases de dados resultaram em um total de 380 artigos. Na etapa de exclusão de artigos duplicados e filtro por ano (2015-2020), restaram 152 artigos, e esse total foi submetido à análise da segunda etapa, que foi exclusão por análise do título, onde restaram somente 126 artigos. Desse total, foi feita a remoção pelo resumo, restando 80 artigos. Depois de realizada a leitura na íntegra restou 16 artigos para o desenvolvimento da presente revisão, como consta no Fluxograma (Figura 1).

Figura 1. Fluxograma de seleção dos artigos para a revisão integrativa

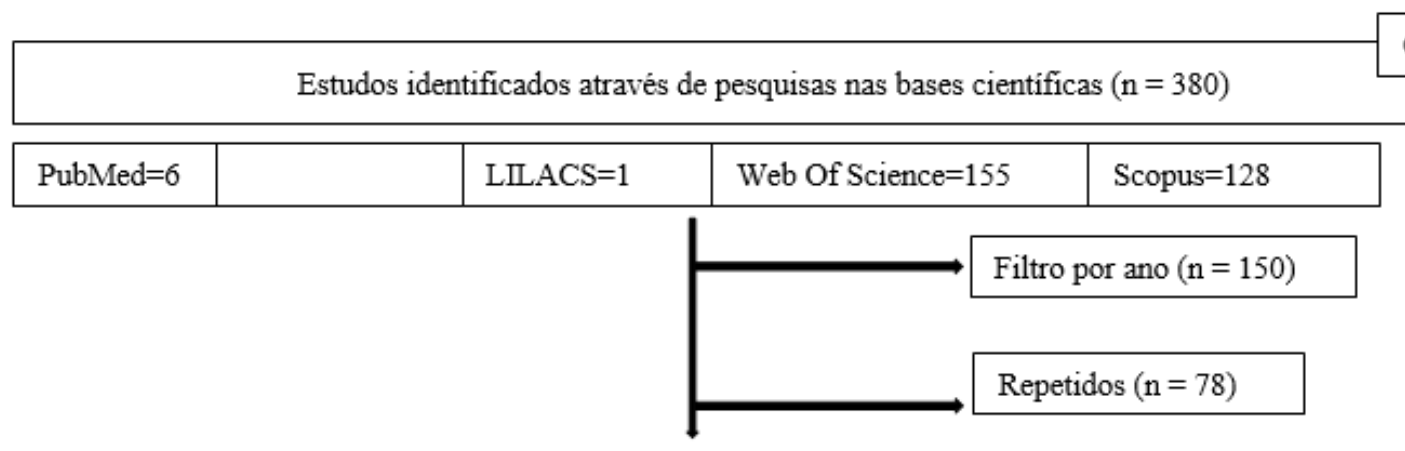

CINAHL $=32$

Resultados após remoção das duplicatas $(\mathrm{n}=152)$

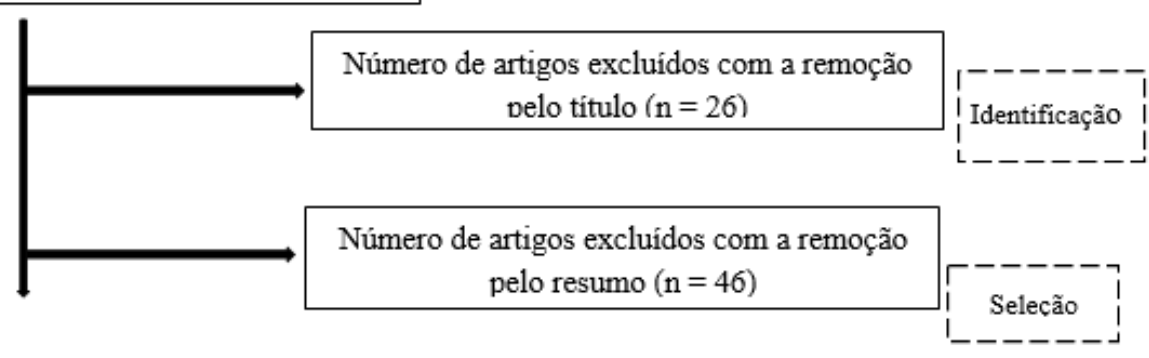

Artigos completos analisados para elegibilidade $(n=80)$

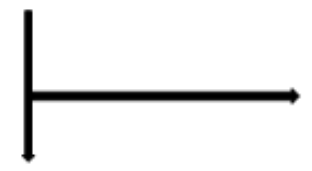

Artigos completos excluídos da análise por não estarem disponiveis na integra $(n=64)$

Estudos incluídos na revisão $(\mathrm{n}=16)$

Fonte: Autores. 


\section{Resultados}

Na presente revisão integrativa, analisou-se 16 artigos que atenderam aos critérios de inclusão e exclusão previamente estabelecidos. Os artigos foram publicados entre os anos 2015 e 2020, cujas pesquisas foram realizadas no Reino Unido (1), Japão (5), Suécia (2), Alemanha (3), Eslovênia (1), França (1), Estados Unidos (2) e Inglaterra (1).

Onze estudos utilizaram exclusivamente a RV (Ambron, 2018; Henriksen, 2017; Ichinose, 2017; Kulkarni, 2020; Nissler, 2019; Osumi, 2017; Osumi, 2019; Perry, 2018; Rutledge, 2019; Snow, 2017; Wake, 2015) para o tratamento e reabilitação de pacientes amputados com Dor do Membro Fantasma (DMF), a partir de instrumentos como: fones de ouvido e Oculus Rift DK2, além de materiais complementares e questionários para coleta dos dados.

E outros utilizaram exclusivamente a RA, os quais utilizaram de materiais como micro-vibradores, faixa elástica, amplificador EMG e vibrador correspondente (Nunzio, 2018; Ortiz-Catalan, 2016; Rothgangel, 2018). Alguns estudos associaram ambas as tecnologias (Ortiz-Catalan, 2016; Lendaro, 2020).

No que se refere ao membro amputado, 12 pesquisaram métodos para membros superiores (Henriksen, 2017; Ichinose, 2017; Kulkarni, 2020; Nissler, 2019; Osumi, 2017; Osumi, 2019; Perry, 2018; Snow, 2017; Wake, 2015; OrtizCatalan, 2016; Nunzio, 2018), somente 02 estudos foram direcionados aos membros inferiores (Ambron, 2018; Rothgangel, 2018) e outros 02 abrangeram tanto membros inferiores, como superiores (Rutledge, 2019; Lendaro, 2020).

Em relação ao tipo de estudo, 04 classificaram-se como estudos de caso (Osumi, 2019; Snow, 2017; Wake, 2015; Lendaro, 2020) outros 07 estudos classificaram-se como estudos experimentais prospectivos longitudinais (Nunzio, 2018; Ambron, 2018; Henriksen, 2017; Ichinose, 2017; Kulkarni, 2020; Rutledge, 2019; Ortiz-Catalan, 2016). Além de outros tipos de metodologias utilizadas como: 01 estudo transversal (Nissler, 2019), 01 estudo quantitativo (Osumi, 2017), 01 análise 01 fatorial exploratória (Osumi, 2019), 01 ensaio clínico (Perry, 2018), 01 estudo controlado randomizado multicêntrico com 3 braços (Rothgangel, 2018).

A maioria dos estudos utilizou como instrumento para coleta de dados o Questionário de Dor McGill de Forma Curta (SF-MPQ) (Osumi, 2017; Osumi, 2019; Perry, 2018; Rutledge, 2019; Snow, 2017; Wake, 2015; Rothgangel, 2018; Osumi, 2020).

Dentre os principais achados, constatou-se, que a associação de RV com estimulação vibrotátil em regiões colaterais da amputação, gerou alívio da DMF (grau de melhora na condição do lado afetado: 22,2\%; condição do ombro do lado afetado: 5,6\%) (Osumi, 2020).

Outro achado importante foi que o uso de RV não gera efeitos colaterais negativos em termos de relatos de dor, mas gera uma leve analgesia, além de comprovar-se a eficácia clínica específica da tarefa de neuro-reabilitação (Snow, 2017; Wake, 2015), bem como a boa aplicabilidade devido ao baixo custo e eficácia (Ambron, 2018).

Em estudos onde foram associadas RV, Realidade Aumentada e reconhecimento de padrões mioelétricos para pacientes amputados de membros superiores e membros inferiores, foi percebido que além da analgesia, foi gerada uma execução do membro fantasma como prática de auto tratamento em suas rotinas diárias, além de melhoria das atividades diárias e qualidade do sono (Ortiz-Catalan, 2016; Lendaro, 2020).

Em pacientes amputados de membros superiores associando a RA e micro-vibradores, um estudo concluiu que o uso de um treinamento sensório-motor multimodal acompanhado da RA, alcançou uma redução significativa na intensidade da DMF durando ao menos 30 dias após o tratamento (Nunzio, 2017).

Houve constatações significativas de reduções significativas na intensidade e percepção da DMF (Rutledge, 2019), conseguindo estabelecer também que a partir da $3^{\mathrm{a}}$ sessão, houve uma diminuição da pontuação da escala de dor, saindo de uma pontuação de 6,11 para 5,56 (Kulkarni, 2020). Em alguns estudos, durante a avaliação de RV foi reportado ausência na sensação de dor (Henriksen, 2017; Ichinose, 2017). 
Em um estudo, percebeu-se que o paciente amputado teve melhor desempenho na utilização do sistema funcional quando comparado aos pacientes não amputados, porém perceberam-se limitações do uso dessas habilidades no cotidiano (Nissler, 2019).

Estudos semelhantes desenvolvidos em períodos diferentes concluíram que houve um alívio satisfatório da DMF, além de restaurar as representações voluntárias do movimento deste membro (Osumi, 2017; Osumi, 2019).

Outro estudo traz que existe a possibilidade do uso do Ambiente de Interação Virtual (AIV) como alternativa de terapia não invasiva e de baixo custo para DMF (Perry, 2018). E em relação a comparação do uso da terapia de espelho com RA e com terapia de espelho tradicional, constatou-se não haver efeitos significativos que diferencie ambas (Rothgangel, 2018). Nos Quadros 1 e 2 estão especificados os respectivos estudos, mediante a terapia virtual utilizada para o manejo da dor fantasma. 
Quadro 1. Síntese dos artigos incluídos na revisão integrativa com utilização da realidade virtual no tratamento da dor fantasma.

\begin{tabular}{|c|c|c|c|}
\hline Autor/Ano/País & Objetivo & Resultados & Conclusões \\
\hline $\begin{array}{l}\text { Ambron, E., et al., } 2018 \\
\text { Estados Unidos }\end{array}$ & $\begin{array}{l}\text { Fornecer evidências adicionais de que o treinamento } \\
\text { do tipo jogo com atividades imersivas de baixo custo } \\
\text { de realidade virtual pode reduzir o PLP em amputados } \\
\text { de membros inferiores. }\end{array}$ & $\begin{array}{l}\text { Ambos os participantes ( } 2 \text { indivíduos) experimentaram uma redução significativa da } \\
\text { dor imediatamente após cada sessão de RV e seus níveis de dor pré-sessão também } \\
\text { diminuíram bastante ao longo do estudo. }\end{array}$ & $\begin{array}{l}\text { Embora preliminares esses dados sustentam a ideia de } \\
\text { que intervenções de RV como a do estudo podem ser um } \\
\text { tratamento eficaz e barato da PLP em amputados de } \\
\text { membros inferiores. }\end{array}$ \\
\hline $\begin{array}{l}\text { Henriksen, B. et al., } 2017 \\
\text { França }\end{array}$ & $\begin{array}{l}\text { Criar uma ilusão de membro recuperado incorporando } \\
\text { significantes tarefas em jogos virtuais que requerem o } \\
\text { uso dos dois membros superiores } \\
\text { simultaneamente. }\end{array}$ & $\begin{array}{l}\text { Dois dos participantes sentiram a ilusão de ter as duas mãos em um alto grau durante } \\
\text { todo o experimento, enquanto o terceiro participante experimentou isso } \\
\text { periodicamente. Os dois participantes, que sentiram essa ilusão, experimentaram } \\
\text { ainda mais que os jogos permitiram que eles usassem seu membro amputado a um } \\
\text { nível alto grau, enquanto participava dos jogos. }\end{array}$ & $\begin{array}{l}\text { Os jogos se mostraram adequados para o treinamento } \\
\text { físico, os participantes puderam jogar até } 90 \text { minutos } \\
\text { durante o dia sem qualquer desconforto significativo que } \\
\text { lhe causasse dor. }\end{array}$ \\
\hline $\begin{array}{l}\text { Ichinose, Akimichi et al., } \\
2017 \\
\text { Japão }\end{array}$ & $\begin{array}{l}\text { Investigar o efeito analgésico produzido pelo feedback } \\
\text { tátil fornecido à bochecha durante a neuro-reabilitação } \\
\text { usando feedback visual. }\end{array}$ & $\begin{array}{l}\text { A taxa média de redução da dor na bochecha } \\
\text { Condição }(33,3 \pm 24,4 \%) \text { foi significativamente maior que na Condição Intacta da } \\
\text { Mão }(16,7 \pm 12,3 \%) \text { e no Estímulo Condição }(12,5 \pm 13,5 \% ; P<0,05) \text {. Mesmo os } \\
\text { pacientes que não sentiram as sensações referidas, relataram redução significativa da } \\
\text { dor após a condição da bochecha. }\end{array}$ & $\begin{array}{l}\mathrm{O} \text { efeito analgésico do feedback visual neuro- } \\
\text { reabilitativo para o membro fantasma o movimento é } \\
\text { significativamente melhorado pela aplicação de feedback } \\
\text { somatossensorial na bochecha do lado afetado. }\end{array}$ \\
\hline $\begin{array}{l}\text { Jai Kulkarni } \text { et al., } 2020 \\
\text { Inglaterra }\end{array}$ & $\begin{array}{l}\text { Avaliar os efeitos de uma atividade de realidade } \\
\text { virtual (VR) no PLP. }\end{array}$ & $\begin{array}{l}\text { Aos } 3 \text { meses de tratamento houve redução na dor média na PLP após a } 3^{\text {a }} \text { sessão de } \\
\text { RV por } 67 \% \text { dos pacientes e esses reportaram redução na intensidade da dor em } \\
\text { virtude de menores NRS (número de episódios de dor). Enquanto aos } 12 \text { meses a } \\
\text { redução da dor média foi de ( } 6,11 \text { v 5,56). Em contrapartida, cinco pacientes } \\
\text { relataram o mesmo NRS (entre } 3-12 \text { meses), enquanto um relatou um aumento na } \\
\text { intensidade de ( } 6 \text { vs.7). }\end{array}$ & $\begin{array}{l}\mathrm{O} \text { uso de um ambiente imersivo de RV pode ter um } \\
\text { pequeno efeito de curto prazo no PLP para a maioria dos } \\
\text { amputados que experimentam PLP. É possível que um } \\
\text { efeito maior possa ser visto se a VR for usada por mais } \\
\text { tempo ou os pontos em que o PLP é mais problemático. }\end{array}$ \\
\hline $\begin{array}{l}\text { Nissler } \text { et al.., } 2019 \\
\text { Alemanha }\end{array}$ & $\begin{array}{l}\text { Investigar como um ambiente de RV pode ser usado } \\
\text { para realizar testes de avaliação e, eventualmente, ser } \\
\text { aplicado para reabilitação de pacientes que sofrem de } \\
\text { mão ou braço imparidades. }\end{array}$ & $\begin{array}{l}\text { O desempenho do usuário da prótese em comparação com os sujeitos especialistas } \\
\text { tem desenvolvimento superior aos pacientes não amputados. Mas, há limitação quanto } \\
\text { a transferência dessas habilidades para o mundo real. Isso pode ser explicado pelo } \\
\text { comportamento às deficiências da prótese. E que um esquema de controle protético } \\
\text { adequado e potencialmente adequado hardware pode solucionar tal questão. }\end{array}$ & $\begin{array}{l}\text { O sistema VITA mostrou aplicabilidade da combinação } \\
\text { de experiência em detecção de intenção com o sistema } \\
\text { VR de última geração para fins de reabilitação. A } \\
\text { ferramenta é facilmente adaptável disponível que } \\
\text { permite simular rápida e realisticamente todo tipo de } \\
\text { problemas do mundo real e exercícios de reabilitação } \\
\text { para pacientes com comprometimentos dos membros } \\
\text { superiores. }\end{array}$ \\
\hline $\begin{array}{l}\text { Osumi } \text { et al., } 2017 \\
\text { Japão }\end{array}$ & $\begin{array}{l}\text { Investigar se a neuro-reabilitação de curto prazo com } \\
\text { um sistema de realidade virtual (RV) restauraria } \\
\text { representações voluntárias de movimento e aliviaria a } \\
\text { dor no membro fantasma (PLP). }\end{array}$ & $\begin{array}{l}\text { Os escores de ambos os questionários sobre a sensação de movimento voluntário do } \\
\text { membro fantasma virtual durante o treinamento de reabilitação de RV foram } \\
\text { significativamente maiores que zero. Após o treinamento de reabilitação de curto } \\
\text { prazo da RV, a pontuação total do SF-MPQ em média em todos os participantes } \\
\text { diminuiu significativamente, além da diminuição significativa do escore NRS. Dois } \\
\text { participantes emergiram como outliers em termos de alívio da dor (Pacientes E e H). } \\
\text { Quando excluídos esses pacientes e repetidas as análises estatísticas, descobrimos que } \\
\text { as três medidas também atingiram significância. }\end{array}$ & $\begin{array}{l}\text { Descobriu-se que a reabilitação de curto prazo da RV } \\
\text { aliviou com sucesso e prontamente o DMF e, } \\
\text { simultaneamente, restaurou as representações voluntárias } \\
\text { do movimento de um membro fantasma, efeito esse que } \\
\text { difere dos efeitos da distração. }\end{array}$ \\
\hline
\end{tabular}




\begin{tabular}{|c|c|c|c|}
\hline $\begin{array}{l}\text { Osumi } \text { et al., } 2019 \\
\text { Japão }\end{array}$ & $\begin{array}{l}\text { Revelar a relação entre efeitos de RV e características } \\
\text { de PLP usando uma análise fatorial exploratória. }\end{array}$ & $\begin{array}{l}\text { A reabilitação VR restaurou significativamente a representação de movimento } \\
\text { quantificada usando o efeito de acoplamento bimanual e aliviou significativamente a } \\
\text { intensidade de DMF (P <0,0001). A análise fatorial revelou que as características da } \\
\text { DMF poderiam ser divididas em dois fatores: "características da dor relacionadas com } \\
\text { somatossensorial" e "características da dor relacionadas à cinestesia". O alívio do } \\
\text { DMF via reabilitação VR foi significativamente correlacionado com "características } \\
\text { da dor relacionadas à cinestesia", mas não "características da dor relacionada à } \\
\text { somatossensorial". }\end{array}$ & $\begin{array}{l}\text { Os presentes resultados sugerem que a variabilidade no } \\
\text { VR o efeito de reabilitação relatada em estudos } \\
\text { anteriores pode ter sido relacionado a diferenças nas } \\
\text { causas do DMF e diferenças nas características do DMF } \\
\text { entre os indivíduos, refletindo os mecanismos } \\
\text { patológicos subjacentes ao DMF. }\end{array}$ \\
\hline $\begin{array}{l}\text { Perry et al., } 2018 \\
\text { Estados Unidos }\end{array}$ & $\begin{array}{l}\text { Explorou-se a possibilidade do Ambiente de } \\
\text { Integração Virtual (VIE), um simulador de membro } \\
\text { superior de realidade virtual, poderia ser usado como } \\
\text { um dispositivo de terapia para tratar efetivamente } \\
\text { DMF em indivíduos com amputação de membro } \\
\text { superior. }\end{array}$ & $\begin{array}{l}\text { Oito homens, militares do serviço ativo com DMF em membro superior foram } \\
\text { recrutados e completaram uma média de } 18,30 \text { minutos de sessões com o VIE. Após } \\
\text { a conclusão das sessões de terapia, houve uma redução significativa no DMF em sete } \\
\text { dos oito ( } 88 \% \text { ) membros afetados e uma redução no PRL em quatro dos cinco }(80 \%) \\
\text { membros afetados. Todos os participantes que negaram a RLP na linha de base }(\mathrm{n}= \\
\text { 3) continuaram a negar a RLP em cada sessão do estudo. }\end{array}$ & $\begin{array}{l}\text { O sucesso do sistema VIE confirma a aplicação como } \\
\text { opção terapêutica não invasiva e de baixo custo, opção } \\
\text { terapêutica DMF para indivíduos com perda do membro } \\
\text { superior para sintomas de DMF e membro fantasma em } \\
\text { indivíduos com perda de membro superior. }\end{array}$ \\
\hline $\begin{array}{l}\text { Rutledge, Thomas et al., } \\
2019 \\
\text { Estados Unidos }\end{array}$ & $\begin{array}{l}\text { Descrever o desenvolvimento de um tratamento RV } \\
\text { para PLP e sensações fantasma e fornecer dados de } \\
\text { viabilidade de testar o tratamento em uma população } \\
\text { de veteranos. }\end{array}$ & $\begin{array}{l}\text { Após o tratamento, } 28,6 \% \text { continuaram relatando sintomas de dor de membro } \\
\text { fantasma e } 28,6 \% \text { relataram sensações fantasmas. Utilidade, realismo, imersão e } \\
\text { satisfação eram uniformemente altas a muito altas. Não houve experiências adversas. } \\
\text { Quatro participantes mostraram melhorias estáveis na intensidade da dor de membro } \\
\text { fantasma e sensações fantasmas e alta avaliação de usuários. }\end{array}$ & $\begin{array}{l}\text { Este estudo de viabilidade de uma nova intervenção de } \\
\text { RV para PLP foi prático e associado com reduções } \\
\text { significativas na intensidade da PLP e sensações } \\
\text { fantasmas. }\end{array}$ \\
\hline $\begin{array}{l}\text { Snow, P. W. et al., } 2017 \\
\text { Estados Unidos }\end{array}$ & $\begin{array}{l}\text { Estabelecer uma estrutura científica mais sólida para } \\
\text { avançar o conhecimento da interação háptica no } \\
\text { tratamento de PLP. }\end{array}$ & $\begin{array}{l}\text { Não há efeitos colaterais negativos em termos de dor relatada. Os resultados iniciais } \\
\text { parecem sugerir níveis mais baixos de dor percebida como resultado da exposição à } \\
\text { intervenção }\end{array}$ & $\begin{array}{l}\text { Resultados parecem fortalecer a incorporação de um } \\
\text { membro virtual pelos participantes proporcionando } \\
\text { efeito háptico na redução dos níveis percebidos de dor. } \\
\text { Embora nenhuma conclusão firme possa ser extraída de } \\
\text { três estudos de casos, parece que um aumento na } \\
\text { incorporação tem um efeito positivo na redução da dor. }\end{array}$ \\
\hline $\begin{array}{l}\text { Wake, N. et al., } 2015 \\
\text { Estados Unidos }\end{array}$ & $\begin{array}{l}\text { Avaliar o efeito clínico imediato deste sistema e } \\
\text { considerar as medidas de protocolo apropriadas de } \\
\text { neuro-reabilitação para o experimento sucessivo. }\end{array}$ & $\begin{array}{l}\text { A taxa máxima de redução da dor foi mostrada em cada caso com a condição tátil. O } \\
\text { escore de dor diminuiu após o treinamento em quatro dos cinco pacientes. Diferentes } \\
\text { condições de feedback tátil foram mais adequadas para diferentes pacientes. }\end{array}$ & $\begin{array}{l}\text { Observamos alívio da dor e eficácia clínica específica da } \\
\text { tarefa para cada paciente. Esses resultados sugerem que } \\
\text { a condição clínica mais adequada será diferente para } \\
\text { cada paciente e que nosso sistema de plataforma pode } \\
\text { oferecer a cada caso o regime mais adequado de maneira } \\
\text { flexível. Nosso sistema, por sua vez, contribuiu para } \\
\text { esclarecer o mecanismo do dor do membro fantasma, } \\
\text { favorecendo o conhecimento clínico. }\end{array}$ \\
\hline
\end{tabular}

Fonte: Autores. 
Quadro 2. Síntese dos artigos incluídos na revisão integrativa com utilização da realidade virtual e aumentada no tratamento da dor fantasma.

Autor/Ano

Ortiz et al., 2016 Suécia

\section{Rothgangel et al., 2018} Holanda

Comparar os efeitos da terapia tradicional com espelhos, um tele tratamento centrado no paciente (PACT) e exercícios sensomotores sem espelho na dor dos membros fantasmas (PLP).

Executar de motor fantasma (PME) facilitada por realidade aumentada/virtual (AR/VR) e séria execução jogos (SG) tem sido proposto como um tratamento para dor nos membros fantasmas (PLP).

De Nunzio, A. M. et al.,

2018

Inglaterra

Osumi et al., 2020

Japão

Explorar o tratamento PLP usando um novo paradigma de treinamento do controle de movimentos de membros fantasmas usando atividade muscular de coto como fonte de feedback visual e tátil.

Utilizar o eletroencefalografia (EEG) para visualizar a ativação sensório-motora através da reabilitação VR combinada com a estimulação vibro tátil em dois pacientes com BPI para os quais o PLP não foi aliviado pela reabilitação simples com VR.

\section{Resultados}

Todos os pacientes referiram uma redução na intensidade e qualidade da do $(51 \%, \mathrm{p}=0,0001) .13$ pacientes relataram que a dor no membro interferia em suas atividades diárias e sono, e após a última sessão do tratamento foi reduzida $(43 \%, p=0,004)$, com redução na dose de ingestão de analgésicos como gabapenfina, pregabalina (redução em $81 \%$ e $33 \%$ ), respectivamente.

Ainda, 12 pacientes tiveram um resultado positivo no índice de classificação da dor, com melhora média relativa e mudança no perfil e intensidade da dor $(56 \%, p=0,001)$. Desses, oito pacientes tiveram uma redução na escala de classificação numérica de pelo menos dois pontos. Enquanto 13 das 15 qualidades diferentes da dor (índice de classificação da dor) obtiveram melhora. Quatro pacientes no grupo terapia de espelho (A e B) e um paciente no grupo controle (C) relataram eventos curtos de DMF aumentado durante o tratamento e dois pacientes do grupo terapia de espelho exibiram graus menores de náusea, reações emocionais e aumento da transpiração no início do tratamento. Houve reduções da frequencia de DMF em todos os grupos, 22 pacientes no gupo reduções da frequênia de DMFento todos os grupos, 22 pacientes no grupo MT e 6 pacientes, e redução também na duração do PLP em 17 pacientes no grupo MT e em 3 pacientes no grupo controle.

Os pacientes fizeram o PME como auto tratamento e incorporaram o dispositivo em suas rotinas diárias. Além disso, os padrões de uso e a adesão à prática de PME foram motivados pela presença de PLP e pela necessidade de seu contexto social. No entanto, as principais dificuldades encontradas foram: tempo e a disponibilidade de eletrodos de uso único.

Uma redução de $32,1 \%$ da intensidade de PLP foi obtida no seguimento (6 semanas após o término do treinamento, com uma redução inicial de 21,6\% imediatamente ao final do treinamento), atingindo efetividade clínica para redução da dor crônica.

A DMF no paciente 1 não se alterou após a reabilitação da RV com estimulação vibro tátil simultânea da mão intacta, porém o uso da reabilitação da RV acompanhada por estimulação vibro tátil do lado ou ombro afetado no lado afetado diminuiu ligeiramente a DMF. No Paciente 2, observamos um alívio da PLP e da coerência das ondas alfa na face afetada e no ombro afetado, mas não na mão intacta.

\section{Conclusões}

O estudo sugere um valor potencial na execução motora do membro fantasma como tratamento para dor no membro fantasma.

Uma intervenção de quatro semanas com terapia de espelho tradicional não forneceu efeitos significativos em comparação com exercícios sensomotores sem espelho na intensidade média, frequência e duração do DMF em quatro semanas. A terapia de espelho tradicional seguida pela terapia de espelho entregue por si mesma alcançou efeitos significativos na de PLP em seis meses em comparação com os grupos controle e tele tratamento.

Boa adesão ao tratamento e, portanto, resultados, podem ser mais aprimorados considerando tipos de usuário diferentes e sers importância da compreensão, de múltiplos ângulos disciplinares, do forte acoplamento e interação entre dor, necessidade percebida e uso de dispositivos médicos na terapia iniciada pelo paciente.

Usando um treinamento sensório-motor multimodal em movimentos de membros fantasmas com recursos visuais e táteis, relatamos uma redução significativa na intensidade do PLP, que durou pelo menos 30 dias após o final do tratamento.

Observamos um efeito analgésico da reabilitação da RV com estimulação vibro tátil simultânea nas condições da face e ombro do lado afetado. 


\section{Discussão}

Os estudos reportaram desfechos positivos quanto a redução da dor no membro fantasma após a utilização instrumentos de realidade virtual e virtual/aumentada, com redução na pontuação numérica na escala de dor, nos pacientes assistidos Kulkarni et al. (2020) e Ortiz-Catalan et al. (2016). Nos estudos de Realidade Virtual (RV) Ambron et al. (2018) e Henrikesen et al. (2017) informaram que esse protocolo pode trazer benefícios e auxiliar no tratamento da dor do membro fantasma' assim como nos estudos de Mercier e Sirigu (2009), Cole et al. (2009), Snow et al. (2017) e Rutledeg et al. (2019), proporciona redução na intensidade e percepção da dor no membro fantasma. Tendo em vista que esse procedimento proporciona redução da dor sentida pelos indivíduos que apresentam essa alteração e pelo fato de encontrarem-se sem dor após realização do tratamento com a RV. E embora esse procedimento apresente pontos positivos deve ser considerado o envolvimento do indivíduo como fator limitante no sucesso do uso dessa realidade. Sendo necessário utilizar atividades divertidas que desperte o interesse dos indivíduos, como organizar quebra-cabeça' Murray et al. (2016) ou jogos de corrida, Ortiz-Catalan et al. (2014) e Ortiz-Catalan et al. (2016).

Além disso, um desses estudos, Lendaro et al. (2020) mencionou melhora quanto a qualidade de sono, redução no uso de medicação para alívio da dor e melhor desempenho nas atividades cotidianas diárias. Mais três estudos, Lendaro et al. (2020), Nissler et al. (2019) e Ichinose et al. (2017) avaliaram positivamente o desempenho dos indivíduos com PLP (dor no membro fantasma), quanto a inserção de dispositivo de RV/AR (realidade virtual e aumentada), a fim de melhorar as atividades cotidianas diárias. Osumi et al. (2019) apresenta o uso da RV combinada com a simulação vibro tátil na geração de um efeito importante na percepção de redução da dor em pacientes com membro fantasma.

Tratando-se da Realidade Aumentada (RA), De Nuzio et al. (2018) comprovou a eficácia do tratamento da dor do membro fantasma através de um novo paradigma de treinamento sensório-motor multimodal (tátil + visual), o qual envolveu movimentos de membros fantasmas através de atividade muscular com recursos visuais e táteis. Sendo a existência de um feedback sensorial multimodal é de extrema importância para o controle da dor do membro fantasma, Brodie et al. (2007). Arango et al. (2013) utilizando RA corrobora com a ideia de que o aumento na proporção de feedback oferecido aos pacientes em atividades de reabilitação que envolvem tecnologias é de suma importância, mas assume a necessidade de imagens que expressem o membro de forma mais realista, com o intuito de potencializar o quadro perceptivo e de reabilitação. Na correlação feedback e Realidade Virtual/Aumentada, Perry et al. (2013) aborda o uso eficaz de um Ambiente de Integração Virtual na analgesia não somente de pacientes que referem a dor no membro fantasma, como também na condição da dor do membro residual, que acomete principalmente militares amputados.

Em estudo anterior, Osumi et al. (2017) também abordou o uso da Realidade Virtual na analgesia da dor do membro fantasma, mas dessa vez concluiu houve melhoria das representações de movimento deste membro, em uma sessão de curta duração. Em um comparativo de tratamentos, Wareham et al. (2020) utilizou a terapia de espelho em militares amputados, também em uma sessão de curta duração, mas não alcançou resultados consideráveis no tratamento da dor do membro fantasma. As diferenças podem-se advir de diferenças nos quadros dos pacientes, como por exemplo, o formato ao qual foi realizada a amputação: cirúrgica ou traumaticamente, dentre outros fatores. Em contra partida, Rothgangel et al. (2018) ao comparar o poder de ação da reabilitação a partir da terapia do espelho, da realidade aumentada e exercícios sensomotores, variando combinações e duração de tratamentos concluiu que o chamado tele tratamento em RA não teve efeitos adicionais em comparação com o tratamento de espelho tradicional auto distribuído, como Wittkopf e Johnson (2017) expõe a terapia de espelho como alternativa eficaz, de baixo custo e sem efeitos colaterais, ou seja, em cenários, a exemplo do Brasil, onde não há o incentivo e/ou adesão do tratamento para dor do membro fantasma, o uso da terapia com espelho tradicional seria uma alternativa de inserção inicial. 
Dessa maneira, fica evidente a contribuição que as referidas realidades podem prover na área de saúde, uma vez que essas são capazes de tornar possível a adaptação do indivíduo quanto às habilidades diárias e minimização da dor, fato tão recorrente no cotidiano desses pacientes. É importante considerar que as amostras que compõem os referidos estudos são pequenas, o que pode ser um fator limitante quanto à confiabilidade dos desfechos. A ausência de recomendações específicas acerca do tema pode ser elucidada como limitação, já que não há consenso quanto o direcionamento das condutas para a temática exposta.

\section{Conclusão}

A presente revisão bibliográfica conduzida neste estudo demonstra que a realidade virtual e aumentada parece ter efeitos benéficos significativos no tratamento da dor fantasma, reduzindo a intensidade e percepção da dor, impactando de forma positiva na qualidade de vida dos pacientes. Contudo, são necessários mais estudos com uma maior amostra e nível de evidência científica, como estudos randomizados controlados. Além disso, recomenda-se que o tratamento seja feito por um período maior para verificar os efeitos da terapia em longo prazo.

\section{Referências}

Akbulut, A., Asci, G., Tarakci, E., Aydin, M. A., \& Zaim, A. (2018). A Wearable Device for Virtual Cyber Therapy of Phantom Limb Pain.

Akbulut, A., Güngör, F., Tarakcı, E., Çabuk, A., \& Aydin, M. A. (2019). Immersive Virtual Reality Games for Rehabilitation of Phantom Limb Pain. Medical Technologies Congress (TIPTEKNO).

Ambron, E., Miller, A., Kuchenbecker, K. J., Buxbaum, L. J., \& Coslett, H. B. (2018). Immersive Low-Cost Virtual Reality Treatment for Phantom Limb Pain: Evidence from Two Cases. Front Neurol. 9:67.

Arango, J. E., Mazo, J. C., \& Palacio, A. P. (2013). Sistema para Rehabilitación del Síndrome del Miembro Fantasma utilizando Interfaz Cerebro-Computador y Realidad Aumentada \%J RISTI - Revista Ibérica de Sistemas e Tecnologias de Informação. 93-106.

Ayoub, A., \& Pulijala Y. (2019). The application of virtual reality and augmented reality in Oral \& Maxillofacial Surgery. BMC oral health. $19(1): 238$.

Brodie, E. E., Whyte, A., \& Niven, C. A. (2007). Analgesia through the looking-glass? A randomized controlled trial investigating the effect of viewing a 'virtual' limb upon phantom limb pain, sensation and movement. Eur J Pain. 11(4):428-36.

Cao, C., \& Cerfolio, R. J. (2019). Virtual or Augmented Reality to Enhance Surgical Education and Surgical Planning. Thoracic surgery clinics. 29(3):329-37.

Cole, J., Crowle, S., Austwick, G., \& Slater, D. H. (2009). Exploratory findings with virtual reality for phantom limb pain, from stump motion to agency and analgesia. Disability and rehabilitation. 31(10):846-54.

Craig, D. M., Emma, P., Stephen, P., Toby, H., Fabrice, C., Jai, K. et al.(2006). Investigating the efficacy of a virtual mirror box in treating phantom limb pain in a sample of chronic sufferers. International Journal on Disability and Human Development. 5(3):227-34.

Demidoff, A. O., Pacheco, F. G., \& Sholl-Franco, A. (2007). Membro-fantasma: o que os olhos não vêem, o cérebro sente. J Ciências \& Cognição. 12:234-9.

Henriksen, B., Nielsen, R., Kraus, M., \& Geng, B. (2017). A virtual reality system for treatment of phantom limb pain using game training and tactile feedback. Virtual Reality International Conference - Laval Virtual, VRIC 2017, Association for Computing Machinery.

Ichinose, A., Sano, Y., Osumi, M., Sumitani, M., Kumagaya, S., \& Kuniyoshi, Y. (2017). Somatosensory Feedback to the Cheek During Virtual Visual Feedback Therapy Enhances Pain Alleviation for Phantom Arms. Neurorehabilitation \& Neural Repair. 31(8):717-25.

Kim, Y., Kim, H., \& Kim, Y. O. (2017). Virtual Reality and Augmented Reality in Plastic Surgery: A Review. Arch Plast Surg. $44(3): 179-87$.

Kulkarni, J., Pettifer, S., Turner, S., \& Richardson, C. (2020). An investigation into the effects of a virtual reality system on phantom limb pain: a pilot study. Br J Pain. 14(2):92-7.

Lendaro, E., Middleton, A., Brown, S., \& Ortiz-Catalan, M. (2020). Out of the Clinic, into the Home: The in-Home Use of Phantom Motor Execution Aided by Machine Learning and Augmented Reality for the Treatment of Phantom Limb Pain. J Pain Res. 195-209.

Mercier, C., \& Sirigu, A. (2009). Training with virtual visual feedback to alleviate phantom limb pain. Neurorehabilitation and neural repair. 23(6):587-94.

Nissler, C., Nowak, M., Connan, M., Büttner, S., Vogel, J., Kossyk, I. et al. (2019). VITA - An everyday virtual reality setup for prosthetics and upper-limb rehabilitation. J Neural Eng. 16(2).

Nunzio, A. M., Schweisfurth, M. A., Ge, N., Falla, D., Hahne, J., Gödecke, K. et al. (2018). Relieving phantom limb pain with multimodal sensory-motor training. J Neural Eng. 2018,15(6):066022. 
Nunzio, A. M., Schweisfurth, M. A., Ge, N., Fella, D., Hahne, J., Godecke, K. et al. (2018). Relieving phantom limb pain with multimodal sensory-motor training. J Neural Eng. 15(6):16.

Ortiz-Catalan, M., Guðmundsdóttir, R. A., Kristoffersen, M. B., Zepeda-Echavarria, A., Caine-Winterberger, K., Kulbacka-Ortiz, K. et al. (2016). Phantom motor execution facilitated by machine learning and augmented reality as treatment for phantom limb pain: a single group, clinical trial in patients with chronic intractable phantom limb pain. Lancet. 388(10062):2885-94.

Ortiz-Catalan, M., Sander, N., Kristoffersen, M. B., Håkansson, B., \& Brånemark, R. (2014). Treatment of phantom limb pain (PLP) based on augmented reality and gaming controlled by myoelectric pattern recognition: a case study of a chronic PLP patient. Front Neurosci. 8:24.

Osumi, M., Ichinose, A., Sumitani, M., Wake, N., Sano, Y., Yozu, A. et al.(2017). Restoring movement representation and alleviating phantom limb pain through short-term neurorehabilitation with a virtual reality system. Eur J Pain. 21(1):140-7.

Osumi, M., Inomata, K., Inoue, Y., Otake, Y., Morioka, S., \& Sumitani, M. (2019). Characteristics of Phantom Limb Pain Alleviated with Virtual Reality Rehabilitation. Pain Med. 20(5):1038-46.

Osumi, M., Sano, Y., Ichinose, A., Wake, N., Yozu, A., Kumagaya, S. I. et al. (2020). Direct evidence of EEG coherence in alleviating phantom limb pain by virtual referred sensation: Case report. Neurocase. 26(1):55-9.

Perry, B. N., Alphonso, A. L., Tsao, J. W., Pasquina, P. F., Armiger, R. S., \& Moran, C. W. (2013). A Virtual Integrated Environment for phantom limb pain treatment and Modular Prosthetic Limb training. 2013 International Conference on Virtual Rehabilitation (ICVR). $26-29$.

Perry, B. N., Armiger, R. S., Wolde, M., McFarland, K. A., Alphonso, A. L., Monson, B. T. et al.(2018). Clinical Trial of the Virtual Integration Environment to Treat Phantom Limb Pain With Upper Extremity Amputation. Front Neurol. 9:770.

Rothgangel, A., Braun, S., Winkens, B., Beurskens, A., \& Smeets, R. (2018). Traditional and augmented reality mirror therapy for patients with chronic phantom limb pain (PACT study): results of a three-group, multicentre single-blind randomized controlled trial. Clin Rehabil. 32(12):1591-608.

Rutledge, T., Velez, D., Depp, C., McQuaid, J. R., Wong, G., Jones, R. C. W. et al. (2019). A Virtual Reality Intervention for the Treatment of Phantom Limb Pain: Development and Feasibility Results. Pain Medicine. 20(10):2051-9.

Snow, P. W., Sedki, I., Sinisi, M., Comley, R., \& Loureiro, R. C. V. (2017). Robotic Therapy for Phantom Limb Pain in Upper Limb Amputees. In: Amirabdollahian F, Burdet E, Masia L, editors. 2017 International Conference on Rehabilitation Robotics. International Conference on Rehabilitation Robotics ICORR. 1019-24.

Uzun, M. (2019). Augmented Reality in Cardiology. The Anatolian Journal of Cardiology. 22.

Wake, N., Sano, Y., Oya, R., Sumitani, M., Kumagaya, S., Kuniyoshi, Y. et al. (2015). Multimodal Virtual Reality Platform for the Rehabilitation of Phantom Limb Pain. 7th International Ieee/Embs Conference on Neural Engineering. International IEEE EMBS Conference on Neural Engineering. 787-90.

Wareham, A. P., \& Sparkes, V. (2020). Effect of one session of mirror therapy on phantom limb pain and recognition of limb laterality in military traumatic lower limb amputees: a pilot study. BMJ military health. 166(3):146-50.

Wittkopf, P. G., \& Johnson, M. I. (2017). Mirror therapy: A potential intervention for pain management \%J Revista da Associação Médica Brasileira. $63: 1000-5$. 